\title{
Tip Growth of Fungal Hyphae
}

\author{
By A. P. J. TRINCI \\ Department of Microbiology, Queen Elizabeth College, \\ Campden Hill Road, London W8 7 AH \\ AND P. T. SAUNDERS \\ Department of Mathematics, Queen Elizabeth College
}

(Received 24 June 1977)

\begin{abstract}
The shapes of tips of rapidly growing hyphae approximate more closely to half ellipsoids of revolution than to hemispheres. It is concluded that provided growth is isotropic the specific rate of wall area expansion will be more nearly proportional to the cotangent of the position angle than to its cosine. Certainly better qualitative fits to the observed data are given by cotangent rather than by cosine curves.
\end{abstract}

\section{INTRODUCTION}

The portion of a hyphal tip involved in extension is called the extension zone. In a hypha growing at a linear rate the shape of this zone, its length and the radius of the hypha at the base of the zone are all constant. These observations imply that both longitudinal and circumferential aspects of hyphal extension are patterned in some stable fashion.

If a tip-growing filament has a hemispherical extension zone in which wall expansion is isotropic and extension zone 'activities' show radial symmetry, then the specific rate of area expansion will be proportional to the cosine of the angle (angle AOP in Fig. I) between the longitudinal axis of the filament and the point on the tip wall (De Wolff \& Houwink, 1954; Green \& King, 1966; Green, 1974). Although Green (1969, I974), Bartnicki-Garcia (1973) and Burnett (I976) have suggested that it may be possible to apply the cosine hypothesis to hyphal extension, no critical analysis has been made of this potential application. This is surprising since a mathematical model which is valid for tip growth would be of considerable value, particularly in studies of hyphal morphogenesis (Da Riva Ricci \& Kendrick, 1972).

In this paper we consider the extent to which hyphae conform to the assumptions implicit in the cosine relationship and, in particular, we assess the importance of tip shape.

We have measured the extension zones of several different hyphae and found that they are not in fact hemispherical but are closer to half ellipsoids of revolution. We then considered what effect this has on Green \& King's result developed for the alga, Nitella, and found that for hyphae it implies that the specific rate of area expansion is approximately proportional to the cotangent of the position angle, rather than to the cosine.

\section{METHODS}

Spores on sterile microscope slides were overlaid with sterile cellophane (P.T. 300, British Cellophane) which in turn was overlaid with a thin (about $\mathrm{I} \mathrm{mm}$ thick) layer of malt extract agar medium (Difco). The slide cultures were incubated in Petri dishes at $25{ }^{\circ} \mathrm{C}$ for $36 \mathrm{~h}$. Hyphae were fixed in Helly's fluid [5\% $(w / v)$ mercuric chloride, $3 \%(w / v)$ potassium dichromate and $0.18 \%(w / v)$ formaldehyde], the tips of leading hyphae were photographed $(\times 400$ magnification) and measurements of their extension zones were made on enlargements ( $\times$ I0) of the negatives using the method of Trinci \& Halford (1975). 
Table I. Extension zones of hyphae growing at linear rates

\begin{tabular}{|c|c|c|c|c|}
\hline Strain & $\begin{array}{l}\text { xtension zone } \\
\text { ength }(a, \mu \mathrm{m})\end{array}$ & $\begin{array}{l}\text { Radius of hypha } \\
\text { at base of } \\
\text { extension zone } \\
(b, \mu \mathrm{m})\end{array}$ & $\begin{array}{l}\text { Ratio } \\
a: b\end{array}$ & 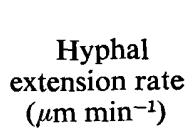 \\
\hline Neurospora crassa SY7A* $^{*}$ & $29 \cdot 2$ & $6 \cdot 55$ & $4 \cdot 46$ & $38 \cdot 0$ \\
\hline Neurospora crassa cot-3* & $25 \cdot 0$ & $6 \cdot 25$ & $4 \cdot 00$ & $18 \cdot 5$ \\
\hline Mucor hiemalis* & $34 \cdot 5$ & $8 \cdot 10$ & $4 \cdot 26$ & $9 \cdot 3$ \\
\hline Trichoderma viride* & $27 \cdot 7$ & $7 \cdot 80$ & $3 \cdot 55$ & I I $\cdot \mathbf{I}$ \\
\hline Botrytis cinerea* & $19 \cdot 6$ & $7 \cdot 90$ & $2 \cdot 48$ & $9 \cdot 6$ \\
\hline $\begin{array}{l}\text { Phycomyces blakesleeanus } \\
\text { sporangiophores } \dagger\end{array}$ & 1800 & 88 & $20 \cdot 45$ & $23 \cdot 3$ \\
\hline Aspergillus giganteus conidiophores & 240 & 25 & $9 \cdot 6$ & $8 \cdot 7$ \\
\hline
\end{tabular}

* Mycelial hyphae. † From Castle (1958). † Trinci \& Banbury (1967).

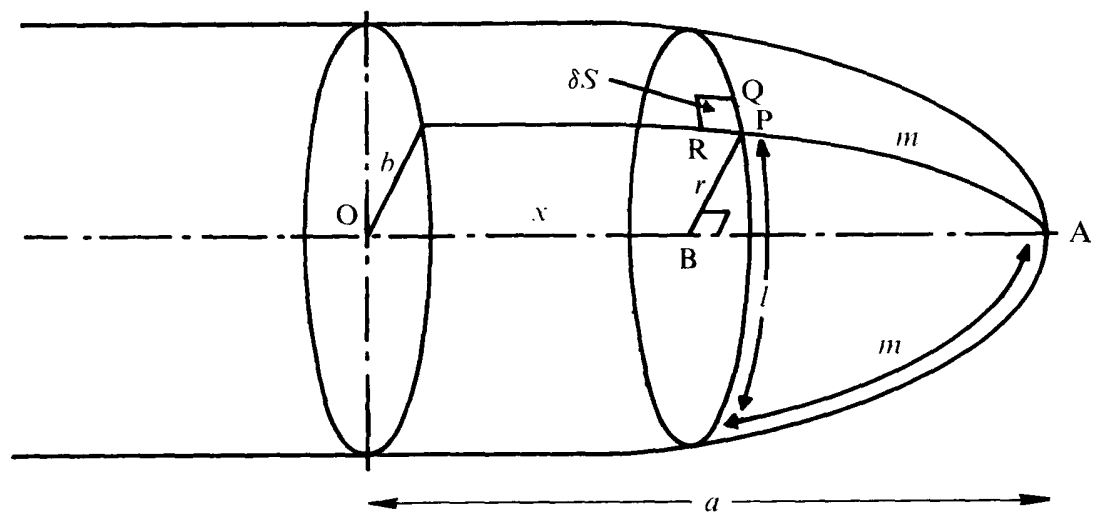

Fig. I. Idealized model of hyphal tip. A, Apex of hypha; O, point on axis at which wall surface area expansion ceases.

\section{RESULTS}

The extension zones of the hyphae examined were not hemispherical in shape (Table I). The ratio of the extension zone length to the radius of the hypha at the base of the extension zone (the ratio $a: b$ in Fig. I) gives an indication of the degree to which the tips deviated from a hemispherical shape. In the following section we consider how tip shape affects the cosine relationship.

\section{CONCLUSIONS}

Green \& King (I966) have derived expressions for the specific area growth rates at different locations on the tips of filaments of Nitella. Their result depends upon the assumption that the tip of a Nitella filament is hemispherical. However, especially in the case of rapid growth (Table I), the tips of fungal hyphae are much more pointed and can be better approximated by half ellipsoids of revolution with a relatively large eccentricity (Reinhardt, I892) than by hemispheres. We shall show that for hyphae this implies that the specific rate of area expansion is more nearly proportional to the cotangent of the position angle than to its cosine.

We begin by considering a tip of circular cross-section but whose shape is otherwise arbitrary. $A$ is the apex of the hypha and $O$ is the point on the axis at which the growth zone ends and the hypha becomes cylindrical. We choose a point $P$ on the tip surface at a distance $m$ from the apex and a distance $l$ around the circumference of the tip from some reference line (Fig. I). We then consider the growth of a small area $\delta S=\delta l \delta m$. 
Let the radius of the tip at $\mathrm{P}$ be $r$. The growth of $\mathrm{PQ}$ is proportional to the growth of the circumference of the tip and since this is, in turn, proportional to the growth of the radius,

$$
\frac{\mathrm{I}}{\delta l} \frac{\mathrm{d}(\delta l)}{\mathrm{d} t}=\frac{\mathrm{I}}{r} \frac{\mathrm{d} r}{\mathrm{~d} t} .
$$

In a short interval of time, $\delta t$, there is an increase in the length PQ given by

$$
\frac{\mathrm{d}(\delta l)}{\mathrm{d} t} \delta t
$$

In the same time interval, the point $\mathrm{P}$ moves a distance

whereas the point $\mathbf{R}$ moves a distance

$$
\frac{\mathrm{d} m}{\mathrm{~d} t}(m) \delta t
$$

$$
\frac{\mathrm{d} m}{\mathrm{~d} t}(m+\delta m) \delta t
$$

so the change in the length of the interval PR is

$$
\frac{\mathrm{d}}{\mathrm{d} m}\left(\frac{\mathrm{d} m}{\mathrm{~d} t}\right) \delta m \delta t \text {. }
$$

The allometric coefficient $K$ is defined as the ratio of the specific growth rates in the two directions, and so

$$
\begin{gathered}
\frac{\mathrm{I}}{\delta m} \frac{\mathrm{d}}{\mathrm{d} m}\left(\frac{\mathrm{d} m}{\mathrm{~d} t}\right) \delta m=K \frac{\mathrm{I}}{\delta l} \frac{\mathrm{d}(\delta l)}{\mathrm{d} t}=\frac{K}{r} \frac{\mathrm{d} r}{\mathrm{~d} t}=\frac{K}{r} \frac{\mathrm{d} r}{\mathrm{~d} m} \frac{\mathrm{d} m}{\mathrm{~d} t} \\
\frac{\mathrm{I}}{\mathrm{d} m / \mathrm{d} t} \frac{\mathrm{d}}{\mathrm{d} m}\left(\frac{\mathrm{d} m}{\mathrm{~d} t}\right)=\frac{K}{r} \frac{\mathrm{d} r}{\mathrm{~d} m} .
\end{gathered}
$$

Hence if $K$ is constant we conclude

where $\mathbf{C}$ is a constant of integration.

$$
\frac{\mathrm{d} m}{\mathrm{~d} t}=\mathrm{Cr} r^{K}
$$

The specific rate of area expansion is the sum of the two specific rates of linear expansion and so is given by

$$
\left(\mathrm{I}+\frac{\mathrm{I}}{K}\right) \frac{\mathrm{d}}{\mathrm{d} m}\left(\frac{\mathrm{d} m}{\mathrm{~d} t}\right)=\left(\mathrm{I}+\frac{\mathrm{I}}{K}\right) \frac{\mathrm{d}}{\mathrm{d} m}\left(\mathrm{Cr}^{K}\right) .
$$

To apply this result we have to know the relationship between $m$ and $r$, and this depends on a knowledge of the actual shape of the tip. Let us suppose that it is close to that of one half of an ellipsoid of revolution of length $a$ and maximum cross-sectional radius $b$. The curve AP is now part of an ellipse whose equation is

$$
\frac{x^{2}}{a^{2}}+\frac{r^{2}}{b^{2}}=\mathrm{I},
$$

where $x$ is the distance from $\mathbf{O}$ to $\mathbf{B}$, the point at which the axis is cut by a plane through $\mathbf{P}$ and perpendicular to the axis.

To find an expression for $\mathrm{d} m / \mathrm{d} t$ it is easiest to take advantage of the fact that the coordinates of any point of this ellipse may be expressed in terms of a single parameter $\alpha$ :

$$
x=a \cos \alpha \quad r=b \sin \alpha .
$$

The arc length $m$ is now given by

$$
m=\int_{0}^{\alpha}\left[\left(\frac{\mathrm{d} x}{\mathrm{~d} \psi}\right)^{2}+\left(\frac{\mathrm{d} r}{\mathrm{~d} \psi}\right)^{2}\right]^{\frac{1}{2}} \mathrm{~d} \psi=\int_{0}^{\alpha}\left[a^{2} \sin ^{2} \psi+b^{2} \cos ^{2} \psi\right]^{\frac{1}{2}} \mathrm{~d} \psi,
$$

where $\psi$ is a dummy variable of integration. Then

$$
\frac{\mathrm{d}}{\mathrm{d} m}\left(\frac{\mathrm{d} m}{\mathrm{~d} t}\right)=\mathrm{C} K r^{K-1} \frac{\mathrm{d} r}{\mathrm{~d} m}=\mathrm{C} K r^{K-1} \frac{\mathrm{d} r}{\mathrm{~d} \alpha} \frac{\mathrm{d} \alpha}{\mathrm{d} m}=\frac{\mathrm{C} K(b \sin \alpha)^{K-1} b \cos \alpha}{\left[a^{2} \sin ^{2} \alpha+b^{2} \cos ^{2} \alpha\right]^{\frac{1}{2}}}
$$




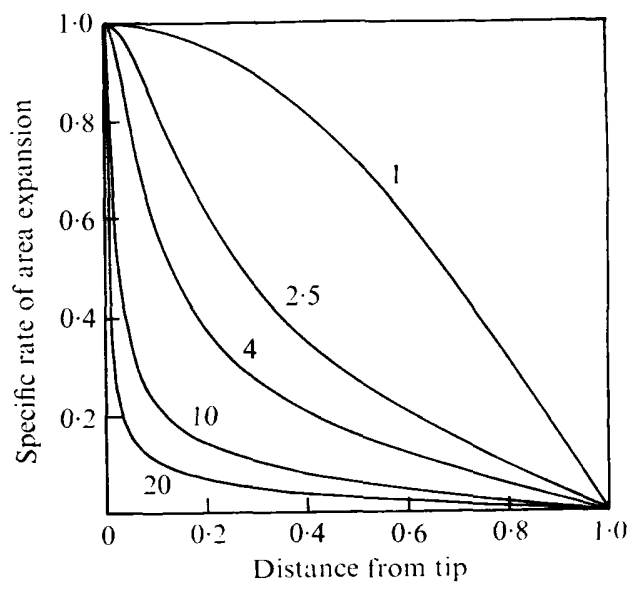

Fig. 2. Specific rate of area expansion as a function of distance from the apex for selected values of the ratio $a: b$ (see Fig. I). For ease of comparison the distance is expressed as a fraction of the total distance from the apex to the end of the extension zone. All distances are measured along the surface of the tip.

In the case of isotropic growth (i.e. if the allometric coefficient is unity) this simplifies to

$$
\frac{\mathrm{d}}{\mathrm{d} m}\left(\frac{\mathrm{d} m}{\mathrm{~d} t}\right)=\frac{\mathrm{C} b \cos \alpha}{\left[a^{2} \sin ^{2} \alpha+b^{2} \cos ^{2} \alpha\right]^{\frac{1}{2}}}
$$

while if we make the further assumption that the tip is hemispherical, then $a=b$, the parameter $\alpha$ is actually the angle AOP, and the above expression reduces to

$$
\frac{\mathrm{d}}{\mathrm{d} m}\left(\frac{\mathrm{d} m}{\mathrm{~d} t}\right)=\frac{\mathrm{C} b}{a} \cos \alpha
$$

which is Green \& King's result. They refer to this quantity as the gradient in the linear expansion and conclude that the gradient in the rate of area expansion is proportional to the cosine of the position; in fact what they calculated was the actual rate of area expansion, which, as we have seen, is the gradient of the velocity.

To see how dependent the result is on the precise shape of the tip, let us suppose that the eccentricity of the ellipsoid is large, so that $a^{2} \gg b^{2}$. In this case we have, except for very small values of $\alpha$,

$$
\frac{\mathrm{d}}{\mathrm{d} m}\left(\frac{\mathrm{d} m}{\mathrm{~d} t}\right) \doteqdot \frac{\mathrm{C} b \cos \alpha}{\left[a^{2} \sin ^{2} \alpha\right]^{\frac{1}{2}}}=\frac{\mathrm{C} b}{a} \cot \alpha,
$$

an entirely different relation. This result is not immediately comparable with equation (I5) because of the approximation involved and because $\alpha$ is in general not the angle AOP, so we have calculated numerically the curves shown in Fig. 2. It is clear from Fig. 2 that tip shape has a profound influence on the nature of the curve generated by such a relationship. It is worth pointing out that the shape of these curves (Fig. 2) is concave upwards, not concave downwards as is the cosine curve. Figure 6D of Chen (1973) suggests that the extension zone of Nitella rhizoids has an $a: b$ ratio (Fig. I) of about $2 \cdot 5$. The data for the meridional and latitudinal specific growth rates obtained by Chen give curves which are very similar in shape to the 2.5 curve in Fig. 2, i.e. concave upwards.

No direct measurements have been made of the specific rate of area expansion of mycelial hyphae. However, Castle (1958) determined the specific growth rates in wall length and girth for stage I sporangiophores of Phycomyces blakesleeanus. His results showed that the allometric coefficient varied between $I$ and 3.5 in the distal half of the extension zone and was below $I$ in the proximal half. Thus longitudinal growth was more rapid than circumferential growth in the distal half whilst the reverse was true in the proximal half. The 


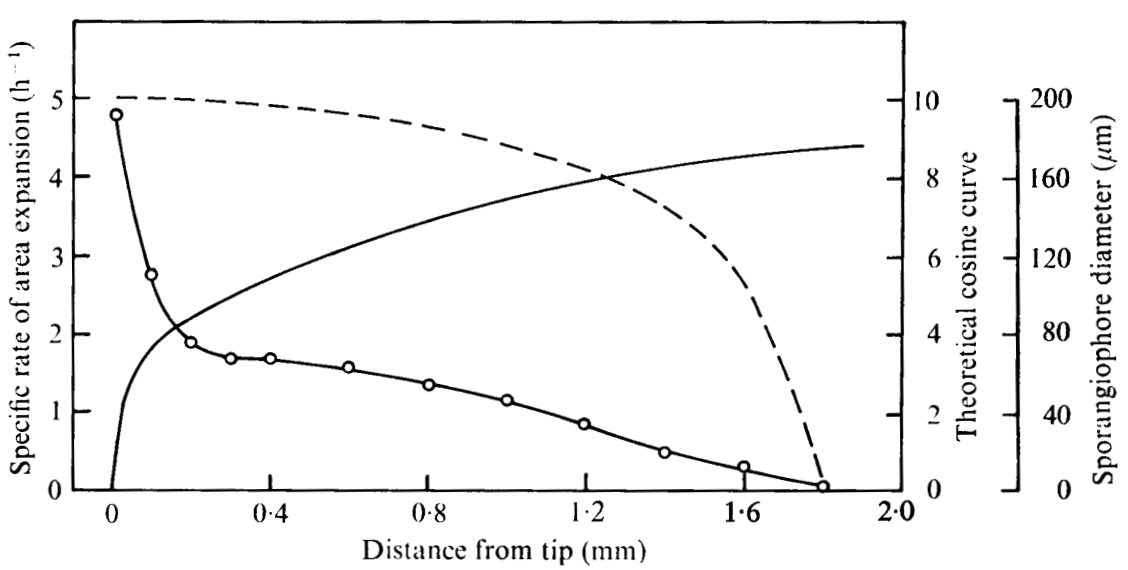

Fig. 3. Hyphal diameter (-) and specific rate of wall area expansion $(O)$ of the extension zone of a stage I sporangiophore of Phycomyces blakesleeanus (calculated from the data of Castle, I958). The hypothetical cosine relationship (--) is also shown.

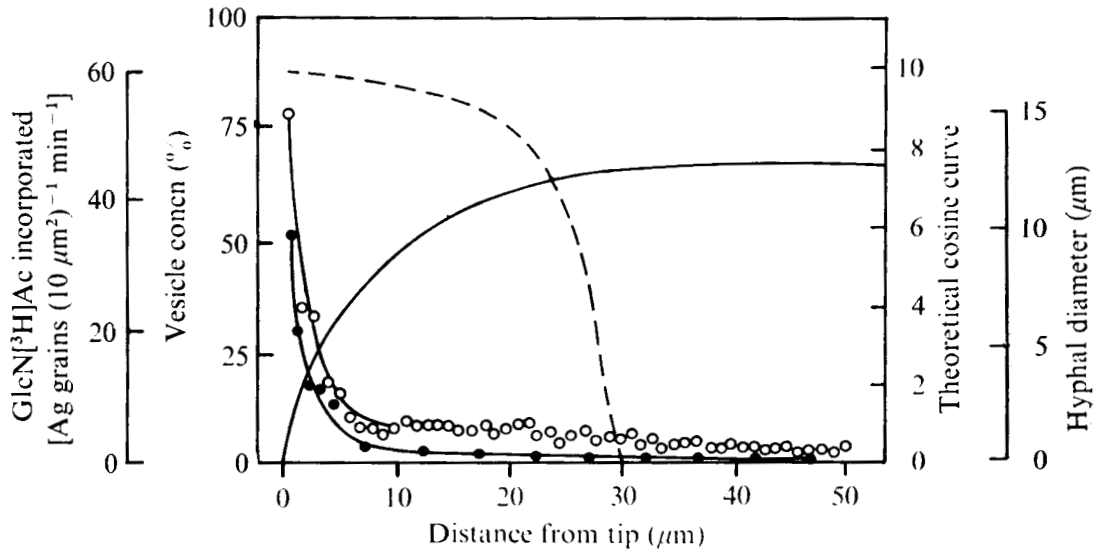

Fig. 4. Hyphal diameter $(-)$ and vesicle concentration $(\bigcirc)$, expressed as the percentage of the protoplasmic volume occupied by vesicles, of Neurospora crassa cot-3 (adapted from Collinge \& Trinci, 1974), and the specific rate of incorporation of $N$-acetyl-D-glucosamine ( $\bullet$ ) of another strain of $N$. crassa, expressed as the number of silver grains per $10 \mu \mathrm{m}^{2}$ resulting from incorporation of $N-\left[{ }^{3} \mathrm{H}\right]$ acetyl-D-glucosamine $\left(\mathrm{GlcN}\left[{ }^{3} \mathrm{H}\right] \mathrm{Ac}\right.$ ) into wall for I min (redrawn from Gooday, 1971). The hypothetical cosine relationship (--) is also shown for the cot-3 hypha.

specific rates of area expansion which we have calculated from Castle's data clearly do not correspond to a cosine relationship (Fig. 3).

The observation that the tip wall of Neurospora crassa does not vary in thickness (Trinci \& Collinge, 1975) means that throughout the extension zone the specific rate of wall synthesis must equal the specific rate of area expansion. Gooday (I97I) has determined the rate of chitin synthesis by following the incorporation of $\mathrm{N}$-acetylglucosamine into the tips of growing hyphae of $N$. crassa. In addition, Collinge \& Trinci (1974) have determined the distribution of vesicles (which supply the membrane and possibly the wall precursors required for tip growth) in the hyphal tips of this same organism. Neither parameter shows a cosine relationship (Fig. 4) but instead, like the Phycomyces data, give curves which are concave upwards, rather than concave downwards.

Values of $a$ and $b$ which correspond to the observed shapes of hyphal tips (Table I) give a reasonable qualitative fit (Fig. 2) to the observed data (Figs 3 and 4). We have not 
considered the effect of varying the allometric coefficient but clearly this will also have a profound effect on the shape of the curve generated from such calculations. Unfortunately it is technically very difficult to obtain this information for mycelial hyphae.

Thus the available evidence suggests that none of the assumptions made in the derivation of the cosine relationship are strictly valid for hyphae. Nevertheless, we have seen that a more accurate (though still somewhat idealized) representation of the shape of the tip improves on Green \& King's result and gives at least qualitative agreement with the observations. We conclude that the basic model of Green \& King (1966) provides a promising starting point for a mathematical analysis of the important problem of tip growth in fungal hyphae.

\section{REFERENCES}

BARTNICKI-GARCIA, S. (1973). Fundamental aspects of hyphal morphogenesis. Symposia of the Society for General Microbiology 23, 245-267.

BURNETT, J. H. (1976). Fundamentals of Mycology, 2nd edn, pp. 6I-69. London: Edward Arnold.

CASTLE, E. S. (1958). The topography of tip growth in a plant cell. Journal of General Physiology 4r, 913-926.

CHEN, C. W. (1973). The kinetics of tip growth in the Nitella rhizoid. Plant and Cell Physiology 14, $63 I-640$.

Collinge, A. J. \& TRINCI, A. P. J. (1974). Hyphal tips of wild-type and spreading colonial mutants of Neurospora crassa. Archives of Microbiology 99, 353-368.

DA Riva Ricci, D. \& Kendrick, B. (1972). Computer modelling of hyphal tip growth in fungi. Canadian Journal of Botany 50, 2455-2462.

De WolfF, P. M. \& Houwink, A. L. (1954). Some considerations on cellulose fibril orientation in growing cell walls. Acta botanica neerlandica 3, 396-397.

GOODAY, G. W. (1971). An autoradiographic study of hyphal growth of some fungi. Journal of General Microbiology 67, 125-133.

Green, P. B. (1969). Cell morphogenesis. Annual Review of Plant Physiology 20, 365-394.

GREEN, P. B. (1974). Morphogenesis of the cell and organ axis - biophysical models. Brookhaven Symposia in Biology 25, 166-190.

Green, P. B. \& KING, A. (1966). A mechanism for the origin of specifically orientated texture in development with special reference to Nitella wall texture. Australian Journal of Biological Sciences 19, 42 I-437.

ReinhardT, M. O. (1892). Das Wachstum der Pilzhyphen. Ein Beitrag zur Kenntniss des Flächenwachstums vegetabilischer Zellmembranen. Jahrbuch für wissenschaftliche Botanik 23, 479-566.

Trinci, A. P. J. \& Banbury, G. H. (1967). A study of the growth of the tall conidiophores of Aspergillus giganteus. Transactions of British Mycological Society 40, 525-538.

Trinci, A. P. J. \& Collinge, A. J. (1975). Hyphal wall growth in Neurospora crassa and Geotrichum candidum. Journal of General Microbiology 9r, 355-361.

Trinci, A. P. J. \& Halford, E. A. (1975). The extension zone of stage I sporangiophores of Phycomyces blakesleeanus. New Phytologist 74, 8 I-83. 